\title{
A Potentially Fatal Complication of Biliary Stent Migration
}

\author{
Jonathan M. Warnock ${ }^{\mathrm{a}, \mathrm{b}}$, Richard J. Thompson ${ }^{\mathrm{a}}$
}

\begin{abstract}
A 77-year-old woman undergoing chemotherapy for mantle cell lymphoma presented with acute lower abdominal pain and pneumoperitoneum. Emergency laparotomy revealed a perforation of the sigmoid colon due to a migrated biliary stent requiring sigmoid resection and colostomy formation. This case highlights the potential complications that may arise from migrated biliary stents and discusses the management options in such patients.
\end{abstract}

Keywords: Biliary stent migration; Sigmoid perforation

\section{Introduction}

This case highights the very rare but very dangerous complication of biliary stent migration. Proximal small bowel perforation by biliary stents has been more widely reported however sigmoid colon perforation is much rarer making this case particularly interesting. Perfortion should be considered in patients with a biliary stent in situ who present with acute abdominal pain and migration of a stent can be identified on plain film abdominal X-ray. We also discuss the potential management options and we have not identified any guidelines on the management of stents that are known to have migrated.

\section{Case Report}

A 77-year-old woman presented with painless obstructive

\footnotetext{
Manuscript accepted for publication September 24, 2012

${ }^{a}$ Department of Surgery, Altnagelvin Area Hospital. Western Health and Social Care Trust. Glenshane Road, Londonderry, BT47 6SB, Northern Ireland

${ }^{\mathrm{b}}$ Corresponding author: Jonathan Warnock, 4 Barnett's Court Mews, Belfast BT5 7FJ, Northern Ireland. Email: johnnyw19@btinternet.com

doi: http://dx.doi.org/10.4021/jmc874w
}

jaundice. She had a background history of diverticular disease. Ultrasound revealed a dilated common bile duct (CBD) with no gallstones. Computed topography showed extensive intra-abdominal lymphadenopathy and soft tissue infiltration around the porta hepatis resulting in biliary obstruction. Subsequent endoscopic retrograde pancreatography was unsuccessful as the ampulla could not be located. A percutaneous transhepatic cholangiogram and rendezvous procedure was therefore performed in order to establish biliary drainage with a plastic biliary stent. Lymph node biopsy revealed mantle cell lymphoma for which she underwent chemotherapy.

After the third cycle of chemotherapy, 2 months after placement of the biliary stent, the patient presented with acute lower abdominal pain and was noted to have free air under the diaphragm. Figure 1 reveals Rigler's sign in keeping with pneumoperitoneum and a migrated biliary stent in the left lower quadrant.

Laparotomy revealed pus in the peritoneum but no gross faecal contamination. A perforation was identified in the sigmoid colon resulting from migration of the biliary stent into the colon with subsequent perforation. A Hartmann's

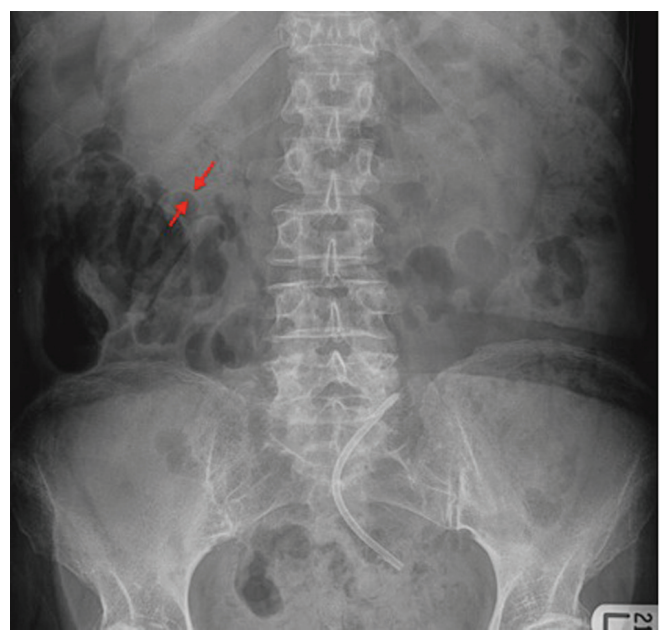

Figure 1. A plain abdominal film. The red arrows demonstrate Rigler's sign caused by free air in the peritoneum. The migrated biliary stent is visible in the pelvis. 


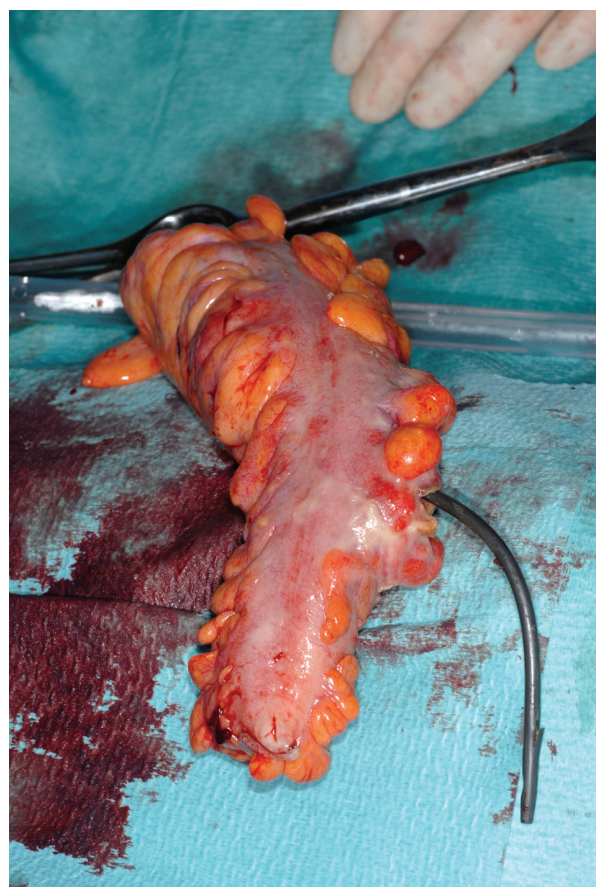

Figure 2. An intra-operative photograph showing the resected segment of colon with the stent perforating through the colonic wall.

procedure was performed. Figure 2 demonstrates the gross specimen of resected colon with the plastic biliary stent visibly perforating through the bowel wall.

\section{Discussion}

Endoscopically inserted biliary stents are used for temporary or permanent relief of obstructive jaundice in a number of conditions such as CBD stones, primary pancreatico-biliary tumours or extrinsic biliary compression caused by porta hepatis lymphadenopathy such as in this case.

The most common complication of a biliary stent is blockage of the stent due to biliary sludge or tumour ingrowth with resulting recurrent obstructive jaundice and cholangitis [1]. Stent migration is another significant complication. In an early study Johanson et al revealed rates of $4.9 \%$ and $5.9 \%$ for proximal or distal stent migration respectively [2]. More recently Arhan et al revealed a stent migration rate of $8.58 \%$ in a heterogeneous group of patients with both benign and malignant disease managed with plastic biliary stents. Stent migration was more common in patients with benign disease [3].

There are several options for dealing with migrated stents. One option is to attempt endoscopic retrieval. A recent review showed that this might be difficult for proximally migrated stents $(71.4 \%$ success) but was more successful for distally migrated stents $(100 \%$ successfully retrieved)
[4]. Indeed $80-86 \%$ success for proximal stent retrieval has also been reported but it is a technical challenge to the endoscopist [5]. A strategy of permitting spontaneous extrusion of a planned temporary stent from the bowel may also be followed. Usually in such cases a delayed x-ray will be undertaken to ensure the stent has successfully been extruded. There have been reported cases of multiple complications due to migrated stents including bowel perforation [1], intraabdominal abscesses [6], fistula formation [7] and bowel obstruction [8].

The duodenum is a common site for perforation due to a migrated biliary stent [9], however complications may arise anywhere along the gastrointestinal tract. A review of the literature in 2007 identified 11 cases of colonic perforation due to migrated stents [1]. It has been postulated that diverticulosis of the colon may increase the risk of perforation of the colon due to a migrated stent becoming impacted in a diverticulum [10]. Other co-morbidities that could lead to stent impaction and subsequent perforation include abdominal hernias and intra-abdominal adhesions [1].

This case highlights that a perforated viscus should be considered in patients with a known biliary stent who present with acute abdominal pain, particularly those with the above co-morbidities. The risk of this complication should be discussed with patients at the time of the insertion. A simple plain abdominal film may clinch the diagnosis as in this case and allow prompt definitive surgical intervention. Stents that are known to have migrated on imaging should ideally be endoscopically retrieved or be closely followed with serial abdominal films to ensure the progression and eventual extrusion of the stent. If the stent fails to progress through the bowel and impaction is suspected then urgent endoscopic retrieval is indicated in order to prevent the potentially fatal complication that occurred in this case.

\section{References}

1. Namdar T, Raffel AM, Topp SA, Namdar L, Alldinger I, Schmitt M, Knoefel WT, et al. Complications and treatment of migrated biliary endoprostheses: a review of the literature. World J Gastroenterol. 2007;13(40):53975399.

2. Johanson JF, Schmalz MJ, Geenen JE. Incidence and risk factors for biliary and pancreatic stent migration. Gastrointest Endosc. 1992;38(3):341-346.

3. Arhan M, Odemis B, Parlak E, Ertugrul I, Basar O. Migration of biliary plastic stents: experience of a tertiary center. Surg Endosc. 2009;23(4):769-775.

4. Katsinelos P, Kountouras J, Paroutoglou G, Chatzimavroudis G, Paikos D, Zavos C, Karakousis K, et al. Migration of plastic biliary stents and endoscopic retrieval: an experience of three referral centers. Surg Laparosc Endosc Percutan Tech. 2009;19(3):217-221. 
5. Lahoti S, Catalano MF, Geenen JE, Schmalz MJ. Endoscopic retrieval of proximally migrated biliary and pancreatic stents: experience of a large referral center. Gastrointest Endosc. 1998;47(6):486-491.

6. Anderson EM, Phillips-Hughes J, Chapman R. Sigmoid colonic perforation and pelvic abscess complicating biliary stent migration. Abdom Imaging. 2007;32(3):317-319.

7. Wilhelm A, Langer C, Zoeller G, Nustede R, Becker H. Complex colovesicular fistula: A severe complication caused by biliary stent migration. Gastrointest Endosc.
2003;57(1):124-126.

8. Garg K, Zagzag J, Khaykis I, Liang H. Surgical relief of small bowel obstruction by migrated biliary stent: extraction without enterotomy. JSLS. 2011;15(2):232-235.

9. Miller G, Yim D, Macari M, Harris M, Shamamian P. Retroperitoneal perforation of the duodenum from biliary stent erosion. Curr Surg. 2005;62(5):512-515.

10. Baty V, Denis B, Bigard MA, Gaucher P. Sigmoid diverticular perforation relating to the migration of a polyethylene endoprosthesis. Endoscopy. 1996;28(9):781. 